\title{
Responses to the Sensory Properties of Fat of Neurons in the Primate Orbitofrontal Cortex
}

\author{
Edmund T. Rolls, ${ }^{1}$ Hugo D. Critchley, ${ }^{1}$ Andrew S. Browning, ${ }^{1}$ Istvan Hernadi, ${ }^{2}$ and Laszlo Lenard ${ }^{2}$ \\ 1 University of Oxford, Department of Experimental Psychology, Oxford OX1 3UD, Great Britain, and 2Department of \\ Physiology, University of Pecs, Pecs H-7643, Hungary
}

\begin{abstract}
The primate orbitofrontal cortex is a site of convergence of information from primary taste, olfactory, and somatosensory cortical areas. We describe the responses of a population of single neurons in the orbitofrontal cortex that responds to fat in the mouth. The neurons respond, when fatty foods are being eaten, to pure fat such as glyceryl trioleate and also to substances with a similar texture but different chemical composition such as paraffin oil (hydrocarbon) and silicone oil $\left.\left[\mathrm{Si}\left(\mathrm{CH}_{3}\right)_{2} \mathrm{O}\right)_{n}\right]$. This is evidence that the neurons respond to the oral texture of fat, sensed by the somatosensory system. Some of the population of neurons respond unimodally to the texture of fat. Other single neurons show convergence of taste inputs, and others of olfactory inputs, onto single neurons that respond to fat. For example, neurons were found that responded to the
\end{abstract}

In previous investigations of the neural bases of taste, olfaction, and feeding in primates (E. T. Rolls, 1994, 1995, 1997, 1999a,b), it has been shown that the primate orbitofrontal cortex contains the secondary taste cortex (Baylis et al., 1994) and that taste neurons found in it (Rolls et al., 1990) only respond to the taste of food when the monkey is hungry (Rolls et al., 1989). There are also connections from the primary olfactory cortex to the orbitofrontal cortex (Carmichael et al., 1994), and the responses of neurons in this secondary and tertiary olfactory cortex represent the reward value of the odor of food, in that the neurons here show olfactory sensory-specific satiety (Critchley and Rolls, 1996a,b; Rolls et al., 1996a; Rolls and Rolls, 1997). The flavor of food is represented and probably formed in this region: in addition to unimodal taste and olfactory neurons in this region, other single neurons respond to both taste and olfactory stimuli (Rolls and Baylis, 1994) and learn olfactory-to-taste associations (Rolls et al., 1996a). In addition to these inputs, there is a somatosensory input to the orbitofrontal cortex from the somatosensory cortex (Carmichael and Price, 1995). Given that the primate orbitofrontal cortex contains a representation of the taste and smell of food, and that this representation is of the reward value of food in that the neural representation is hunger dependent and in that brainstimulation reward of the primate orbitofrontal cortex is hungerdependent (Mora et al., 1979, 1980; Rolls, 1999a), we performed the experiments described here to investigate whether there is a

Received Sept. 25, 1998; revised Dec. 1, 1998; accepted Dec. 7, 1998.

This research was supported by Medical Research Council Grant PG8513790 to E.T.R., and by a British Council/Hungarian Academy of Sciences grant to L.L. and E.T.R.

Correspondence should be addressed to Professor E. T. Rolls, University of Oxford, Department of Experimental Psychology, South Parks Road, Oxford OX1 3UD, Great Britain.

Copyright (C) 1999 Society for Neuroscience $\quad 0270-6474 / 99 / 191532-09 \$ 05.00 / 0$ mouth feel of fat and the taste of monosodium glutamate (both found in milk), or to the mouth feel of fat and to odor. Feeding to satiety reduces the responses of these neurons to the fatty food eaten, but the neurons still respond to some other foods that have not been fed to satiety. Thus sensory-specific satiety for fat is represented in the responses of single neurons in the primate orbitofrontal cortex.

Fat is an important constituent of food that affects its palatability and nutritional effects. The findings described provide evidence that the reward value (or pleasantness) of the mouth feel of fat is represented in the primate orbitofrontal cortex and that the representation is relevant to appetite.

Key words: fat; food; mouth; oral; orbitofrontal cortex; olfaction; taste; hunger; satiety

representation of the sensory properties of the important macronutrient fat in the primate orbitofrontal cortex.

Fat is an important component of normal food intake in primates, and it is palatable. Humans are natural omnivores, who like macaques have evolved to take in a diet (after weaning) that consists of vegetable matter supplemented by protein-rich and fat-rich food sources that are a valuable source of essential amino acids and essential fats. Mechanisms that sense and regulate the dietary intake of fat are of great importance clinically. In particular, high dietary fat intake is strongly implicated in the etiology of cardiovascular morbidity and deaths. Studies of satiety in normal human subjects indicate that high-fat foods may not produce satiety or reduce hunger ratings to the same degree as isocaloric high-carbohydrate or high-protein foods, and that foods containing fat may be overeaten in terms of and because of their high energy density (Johnson and Vickers, 1993; Warwick et al., 1993; B. J. Rolls, 1995; Bell et al., 1998). This indicates that the mechanisms controlling the intake of fats might be less sensitive than those controlling protein or carbohydrate intake. Together these data indicate that fat in the diet may help to make food palatable, and that because of its energy density it may be important to actively monitor fat intake. The research described in this paper investigates the mechanisms that sense oral fat and the neural representation of oral fat, of its palatability, and how that representation is affected by satiety.

\section{MATERIALS AND METHODS}

Recordings. Recordings were made from single neurons in the orbitofrontal cortex, which included both the medial and lateral areas in which taste and olfactory responses have been described previously (Rolls and Baylis, 1994; Rolls, 1997). The subjects were three rhesus macaques (Macaca mulatta) weighing $2.5-3.5 \mathrm{~kg}$. Neurophysiological methods were the same as described previously (Rolls, 1976; Scott et al., 1986a,b; Rolls et al., 
1990; Yaxley et al., 1990; Rolls and Baylis, 1994). All procedures, including preparative and subsequent ones, were performed in accordance with the "Policy on the Use of Animals in Neuroscience Research" of the Society for Neuroscience and were licensed under the United Kingdom Animals (Scientific Procedures) Act 1986. Each monkey was fed during the experiments and on return to the home cage and was allowed ad libitum access to water. Glass-coated tungsten microelectrodes were constructed in the manner of Merrill and Ainsworth (1972) without the platinum plating. A computer (Pentium) with real-time digital and analog data acquisition collected spike arrival times and displayed on-line summary statistics or a peristimulus time histogram and rastergram. To ensure that the recordings were made from single cells, the interspike interval was monitored continuously to make sure that intervals of $<2 \mathrm{msec}$ were not seen, and also the waveform of the recorded action potentials was monitored continuously using an analog delay line.

Localization of recordings. X-radiography was used to determine the position of the microelectrode after each recording track relative to permanent reference electrodes and to the anterior sphenoidal process. This is a bony landmark whose position is relatively invariant with respect to deep brain structures (Aggleton and Passingham, 1981). Microlesions made through the tip of the recording electrode during the final tracks were used to mark the location of typical units. These microlesions together with the associated x-radiographs allowed the position of all cells to be reconstructed in the $50 \mu \mathrm{m}$ brain sections with the methods described in Feigenbaum and Rolls (1991).

Screening of neurons. Orbitofrontal cortex cells were tested for their responsiveness to taste, olfactory, and visual stimuli. The gustatory stimuli that were used included $1.0 \mathrm{M}$ glucose, $0.1 \mathrm{M} \mathrm{NaCl}, 0.01 \mathrm{M} \mathrm{HCl}$, $0.001 \mathrm{M}$ quinine- $\mathrm{HCl}$, and $0.1 \mathrm{M}$ monosodium glutamate (MSG). The concentrations of most of the tastants were chosen because of their comparability with our previous studies, and because they are in a sensitive part of the dose-response curve. The monkey's mouth was rinsed with distilled water during the intertrial interval (which lasted at least $30 \mathrm{sec}$, or until neuronal activity returned to baseline levels) between taste stimuli. The stimuli within a set were delivered in random sequence. The stimuli were delivered by mouth in quantities of $0.2 \mathrm{ml}$ with a hand-held $1 \mathrm{ml}$ syringe. For chronic recording in monkeys, this manual method for stimulus delivery is used because it allows for repeated stimulation of a large receptive surface despite different mouth and tongue positions adopted by the monkeys (Scott et al., 1986a,b). The firing rates were measured in a $3 \mathrm{sec}$ poststimulus delivery period, because this is the period in which taste neurons, and the neurons described here, were found to have their main responses. For additional comparisons, the neuronal responses were also tested to a range of foods including banana, orange, apple juice, milk, and $20 \%$ blackcurrant juice.

To test for the oral effects of fat on neuronal activity, a set of fat and fat-related stimuli were delivered in the same way with a pseudorandom sequence. The fat stimuli included "single" cream (cream) (18\% fat), "double" cream (47.5\% fat), triolein, groundnut oil, and "half-fat" milk (milk) (1.8\% fat, which is categorized as low fat in the United States). (For comparison, "skimmed milk" contains $0.1 \%$ fat.) These were used to examine whether fat is represented in the responses of cortical (taste) neurons. Cream was used because it was found to be a palatable high-fat food, in that it was readily ingested by monkeys compared with vegetable oil, lecithin, and vegetable oil or lecithin emulsions. Half-fat milk $(1.8 \%$ fat) and skimmed milk ( $0.1 \%$ fat) were used to investigate whether the neurons would respond to lower concentrations of fat. [We note that macaques are omnivorous; after weaning they very readily accept milkbased products, and lactose intolerance is rare (Streett and Jonas, 1980; Benno et al., 1987).] Triolein (glyceryl trioleate) was used as a pure fat. Vegetable oil (59.5\% monounsaturates, 34\% polyunsaturates, and $6.5 \%$ saturates) and groundnut oil were used as other natural high-fat stimuli. To investigate whether the neurons responsive to cream were in some way responding to the somatosensory sensations elicited by the fat, stimuli with a similar mouth feel but nonfat chemical composition were used. These stimuli included paraffin oil (pure hydrocarbon) and silicone oil $\left.\left[\mathrm{Si}\left(\mathrm{CH}_{3}\right)_{2} \mathrm{O}\right)_{\mathrm{n}}\right]$. The viscosities of the stimuli [expressed in centipoise (cP) for which values of 1-1.5 were obtained for tap water] were as follows: $1.8 \%$ fat milk, $3.6 \mathrm{cP} ; 18 \%$ cream, $65 \mathrm{cP}$; vegetable oil, $67 \mathrm{cP}$; triolein, $71 \mathrm{cP}$; paraffin oil, $175 \mathrm{cP}$; and silicone oil, $280 \mathrm{cP}$. To control for specificity of the somatosensory input that could activate these neurons, other nonfat-related, oral somatosensory, or motor responsiveness of neurons was screened for by allowing the monkey to chew on a short length of plastic tubing. Because of the tenacious nature of the oral coating resulting from the delivery of cream or oil, the interstimulus interval was prolonged (usually $>2 \mathrm{~min}$ ) and repeated rinses with water were given during this period.

Responses to odorants were determined either using a perfumer strip method or an olfactory discrimination task (Critchley and Rolls, 1996a,b; Rolls et al., 1996a). The criteria for olfactory responsiveness were a significant elevation of cellular firing above the spontaneous firing rate to an odorant (measured during a $5 \mathrm{sec}$ period of presentation in front of the monkey's nose of a cotton bud/perfumer strip saturated in odor vapor) and no response to an odorless cotton bud used as a control. The olfactory discrimination task involved the randomized delivery of odorant-saturated air via a computer-driven olfactometer (Critchley and Rolls, 1996a). A cue tone preceded the delivery, after which the monkey was required to sample each odor to identify odors as part of a Go/NoGo task. A lick response to a rewarded odorant was rewarded with the delivery of a sweet aspartame solution from the lick tube; a lick response on the NoGo trials was associated with the delivery of a mildly aversive saline solution. On-line rastergrams and statistics enabled the determination of olfactory responsiveness. An air extraction apparatus was located above the monkey's head to remove odor (Critchley and Rolls, 1996a).

Satiety experiments. At the start of the experiments on each day, the monkey was hungry, having been fed $\sim 14$ hr previously. Satiety experiments were performed on cells having effective and reliable responses to the mouth feel of fat. The responses of the neuron to a subset of pure tastants (and to natural food odors if the cell had an olfactory response) were measured before and after satiation. In most cases, single cream was used to produce satiety, and the corresponding test stimulus was the same cream. In some of the experiments it was possible to record the responses of the cells to the cream at intermediate stages of satiation. For each stimulus, between 4 and 10 trials were performed to ensure statistical validity. Aliquots of $10 \mathrm{ml}$ of the satiating solution were fed to the monkey, during which the behavioral response to the solution was observed. The behavioral acceptance of this solution was rated according to the criteria used previously (Rolls et al., 1989). Scores on the scale of acceptance or rejection were based on the following behavioral criteria: $+2.0=$ maximal acceptance, reaching for the solution with hands and mouth, avid licking; $+1.0=$ clear acceptance, opening the mouth, licking, and swallowing the solution; $0.0=$ neutrality, swallowing the solution when placed in the mouth, absence of avidness, and no attempt made to obtain the solution; $-1.0=$ clear rejection, pursing the lips to prevent administration of the solution, and failure to swallow all of the solution placed in the mouth; $-2.0=$ maximum rejection, pursing the lips and closing the teeth, using the tongue to eject delivered solution, swallowing little, and using the hands to push away the solution. If the behavior was intermediate between these types, then intermediate scores were given.

\section{RESULTS}

The data described here were obtained during 283 recording tracks in three monkeys. Out of 1145 neurons in the orbitofrontal region tested for gustatory responses, 11 neurons $(0.96 \%)$ had responses to fat stimuli (10 to fat in the mouth and 1 to the odor of cream). The differential effect was confirmed statistically by one-way ANOVAs of the neuronal responses across a range of stimuli that typically included fat stimuli such as cream and of the neuronal responses to the tastants [i.e., glucose, $\mathrm{NaCl}, \mathrm{HCl}$, MSG, and quinine] (Rolls et al., 1996b), and by subsequent post hoc Dunnett's analyses showing a significant elevation of firing rate relative to the spontaneous rate to at least one of the fat-related stimuli at $p<0.001$ or less. An additional criterion to having a significant response in the ANOVAs was that the neuron should not respond to mouth movements produced by chewing a nonfood, nonfat stimulus (see Materials and Methods). The neurons that responded to fat had a range of stimuli to which they responded best, including glucose, $\mathrm{NaCl}, \mathrm{MSG}$, water, and cream, and complex foods such as apple and banana. Examples of the responses of these neurons are described next, and then a summary of the properties of the population of neurons analyzed is given. 
Figure 1. Responses of a primate orbitofrontal cortex neuron (be0511) to fat in the mouth and to stimuli with a similar texture but not to taste. The mean response of the neuron and the SEM are shown. The spontaneous firing rate is shown. The fat stimuli were cream (47.5 and $18 \%$ ), milk containing $1.7 \%$ fat, triolein, and vegetable oil. The chemically different stimuli with a similar texture were silicone oil $\left.\left[\mathrm{Si}\left(\mathrm{CH}_{3}\right)_{2} \mathrm{O}\right)_{n}\right]$ and paraffin oil (hydrocarbon). The taste stimuli were $1.0 \mathrm{M}$ glucose $(G l u c), 0.1 \mathrm{M} \mathrm{NaCl}, 0.01 \mathrm{M} \mathrm{HCl}, 0.001 \mathrm{M}$ quinine- $\mathrm{HCl}$ (Q), $0.05 \mathrm{M}$ monosodium glutamate $(M S G)$, and distilled water $\left(\mathrm{H}_{2} \mathrm{O}\right)$.

Figure 2. Responses of a primate orbitofrontal cortex neuron (be047) to fat in the mouth, to stimuli with a similar texture, and to the taste of MSG. Conventions as in Figure 1.
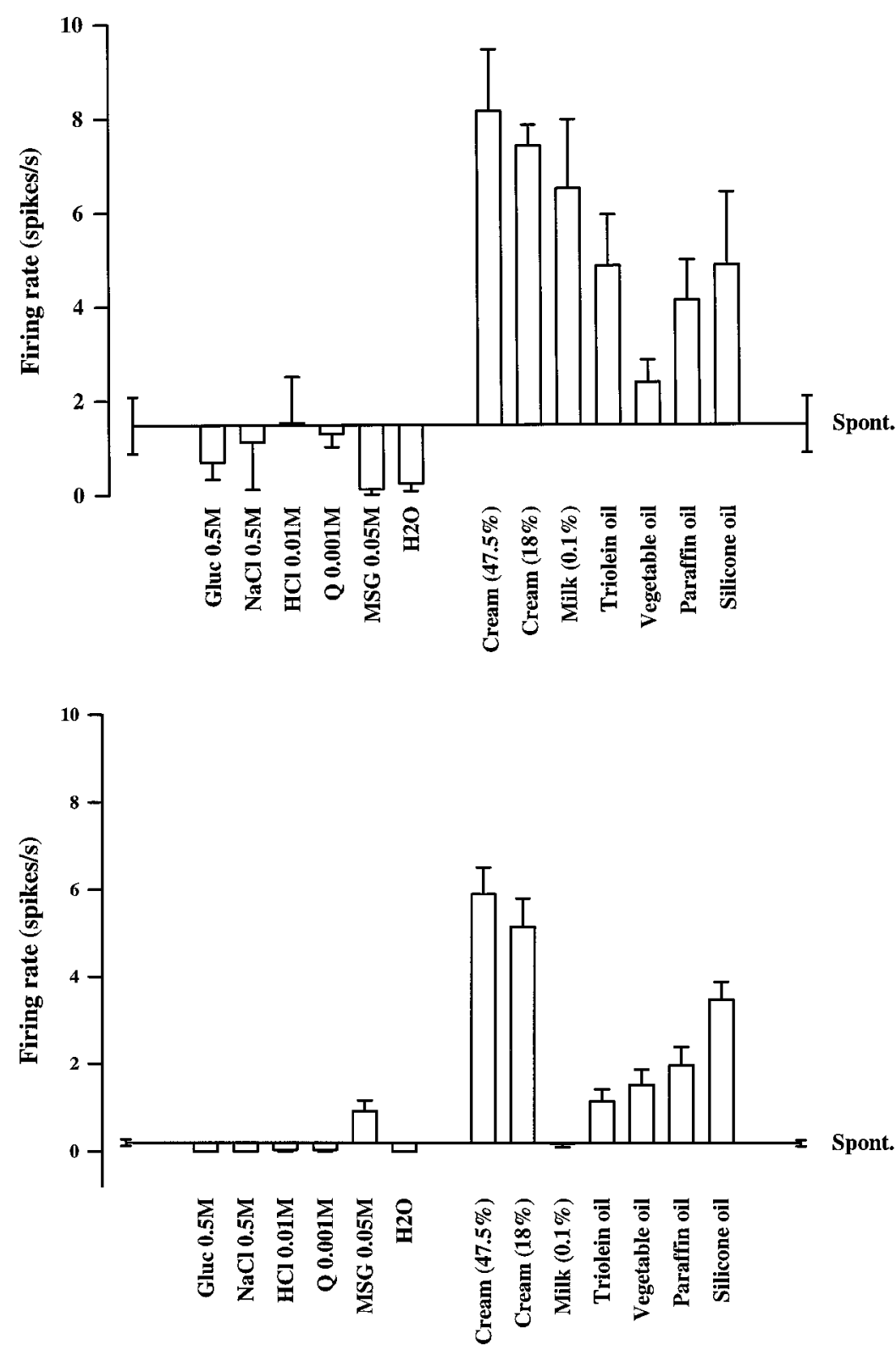

The responses of an example of a neuron (be0511) that responded to fat in the mouth but not to taste stimuli are shown in Figure 1. The neuron responded to cream with $47.5 \%$ or $18 \%$ fat. The neuron also increased its firing rate to a pure fat (triolein) and a natural fat (vegetable oil). The neuron also responded to substances that are not chemically fat but had a similar texture, namely paraffin oil (pure hydrocarbon) and silicone oil $\left.\left[\mathrm{Si}\left(\mathrm{CH}_{3}\right)_{2} \mathrm{O}\right)_{n}\right]$. In a preplanned ANOVA to test its responsiveness to cream $(47.5 \%$ and $18 \%)$, fat (triolein and vegetable oil), and substances with a fat-like texture (silicone oil and paraffin oil), the ANOVA was significant $(p<0.001)$, and the post hoc Dunnett's test showed that there was a significantly increased firing rate to cream $(p<0.001)$ and the fat-like texture as a combined group $(p=0.012)$.

An example of a bimodal neuron that responded both to the texture of fat in the mouth and to taste is shown in Figure 2 (be047). The neuron responded to cream (47.5\% and $18 \%$ fat; see below for statistics). The neuron responded to pure fats (triolein and vegetable oil; see below), and its response was based on texture: its firing rate also increased to paraffin oil and to silicone oil, the nonfat substances with a fat-like texture. The neuron also had a taste response, in that it responded to $0.05 \mathrm{~m}$ monosodium glutamate. [This was shown statistically to be a taste response, in that there was a significant one-way ANOVA result performed across the tastants only (i.e., glucose, $\mathrm{NaCl}, \mathrm{HCl}, \mathrm{MSG}$, and quinine), showing differential responses across the set of tastants, and by a subsequent Dunnett's test showing a significant response to monosodium glutamate; $p<0$.001.] In a preplanned ANOVA followed by a post hoc Dunnett's test with the spontaneous rate as the comparison condition, to test its responsiveness to fats and related stimuli, it was found that the ANOVA was significant and that the neuron had significant responses to cream $(47.5 \%$ and $18 \% ; p<0.001$ for the combined group), fat (triolein and vegetable oil; $p<0.05$ for the combined group), and substances with a fat-like texture (silicone oil and paraffin oil; $p<0.001$ for the combined group).

An example of another neuron with bimodal taste and fat responses, to illustrate broader tuning to taste, is shown in Figure 


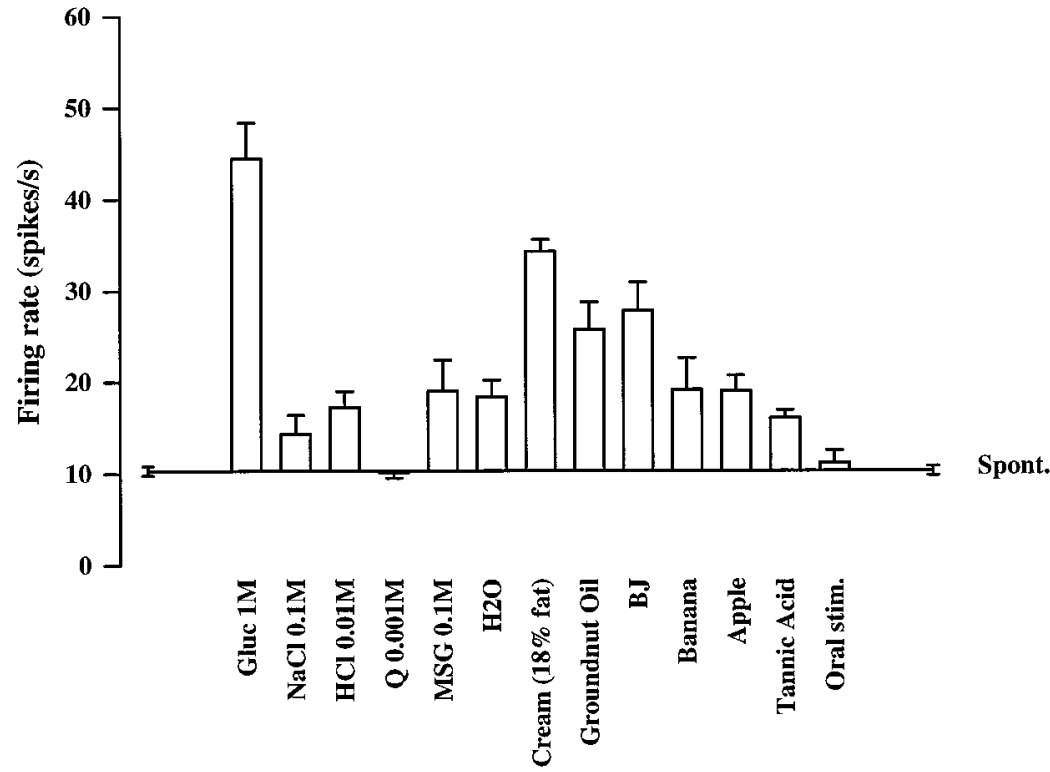

Figure 3. Responses of a primate orbitofrontal cortex neuron (au143a) to fat in the mouth, to stimuli with a similar texture, and to some of the taste stimuli. Conventions as in Figure 1. Responses to tannic acid $(0.001 \mathrm{M})$, blackcurrant juice $(B J)$, and eating pieces of banana and apple are also shown. Oral stim., Mechanical stimulation (see Materials and Methods); Spont., spontaneous firing rate.
3 (au143a). The neuron also increased its firing rate to the mouth feel of astringency produced by tannic acid, which is sensed through the somatosensory system (Critchley and Rolls, 1996c).

An example of a neuron that responded to the odor of cream is shown in Figure 4 (au142b). Before the monkey was fed to satiety, the neuron responded to the odors of cream, apple, and banana, but not to the odor of caprylic acid (plastic). The monkey was then fed to satiety with cream, of which he consumed $80 \mathrm{ml}$. The rating for the acceptance of cream changed from +2 to -2 . After satiety, the neuron no longer responded to the odor of cream but did respond to the odors of other foods (which remained acceptable to the monkey). There was a significant interaction $(p<$ 0.002) in a two-way ANOVA in which the factors were odor type (cream, apple, and banana), and pre-satiety versus post-satiety. Thus sensory-specific satiety for cream reflected the sensoryspecific decrease in the response of this neuron to the odor of cream.

Sensory-specific satiety-related decreases in neuronal responses were also found for neurons responding to the texture of fat. An example is shown in Figure 5 (au159). The neuron responded to taste (with a best response to acid) and also to fat (tested with cream and groundnut oil). After feeding to satiety with cream, the response of the neuron decreased to cream in the mouth but not significantly to groundnut oil (as shown by a two-way ANOVA; $p<0.02$ ). This is an indication that even between fats there can be sensory-specific satiety effects, although these could be contributed to by the taste inputs received by this neuron. After feeding to satiety with cream, there were even small increases to some stimuli, as is common with sensory-specific satiety at both the neuronal level in macaques and the behavioral level in humans (Rolls and Rolls, 1997; Rolls, 1999a,b).

To extend the ecological validity of the findings described for this new class of neuron in the orbitofrontal cortex described here, we investigated whether this type of neuron would respond to the fat in other types of fatty food. In particular, the fat and fat-related stimuli described so far were liquid, and it was of interest to investigate whether the neurons would respond to fatty solid foods such as nuts. Such testing is illustrated for neuron bb027 in Figure 6. The neuron responded to fats or fat-related stimuli (e.g., cream, vegetable oil, and paraffin oil) and had a
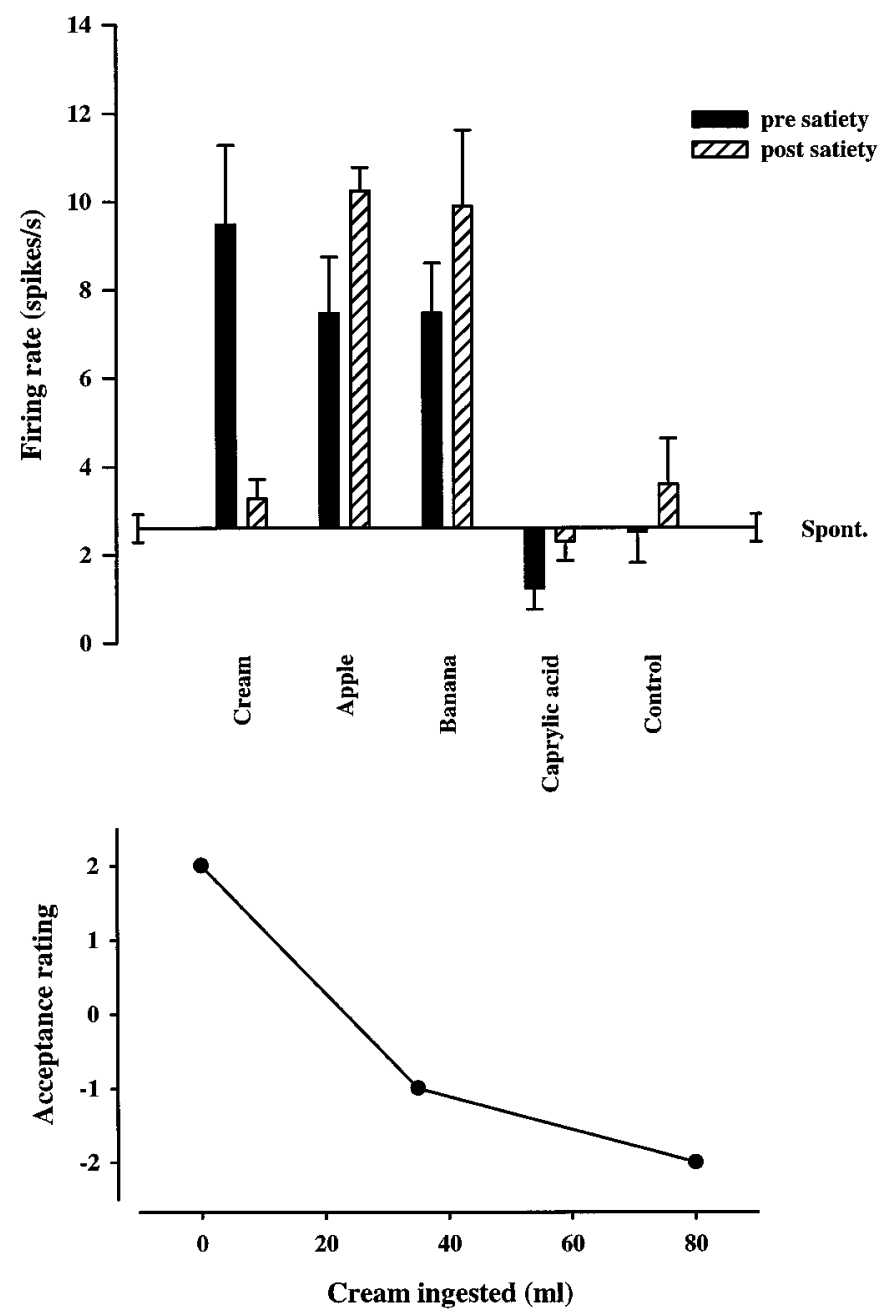

Figure 4. Responses of a primate orbitofrontal cortex neuron to the odor of some foods, including that of cream, apple, and banana. The neuron did not respond to the odor of caprylic acid. After feeding to satiety with $80 \mathrm{ml}$ of cream, the rating for the acceptance of cream changed from +2 to -1 (see below), and the neuron responded much less to the odor of cream. Other conventions as in Figure 1. 

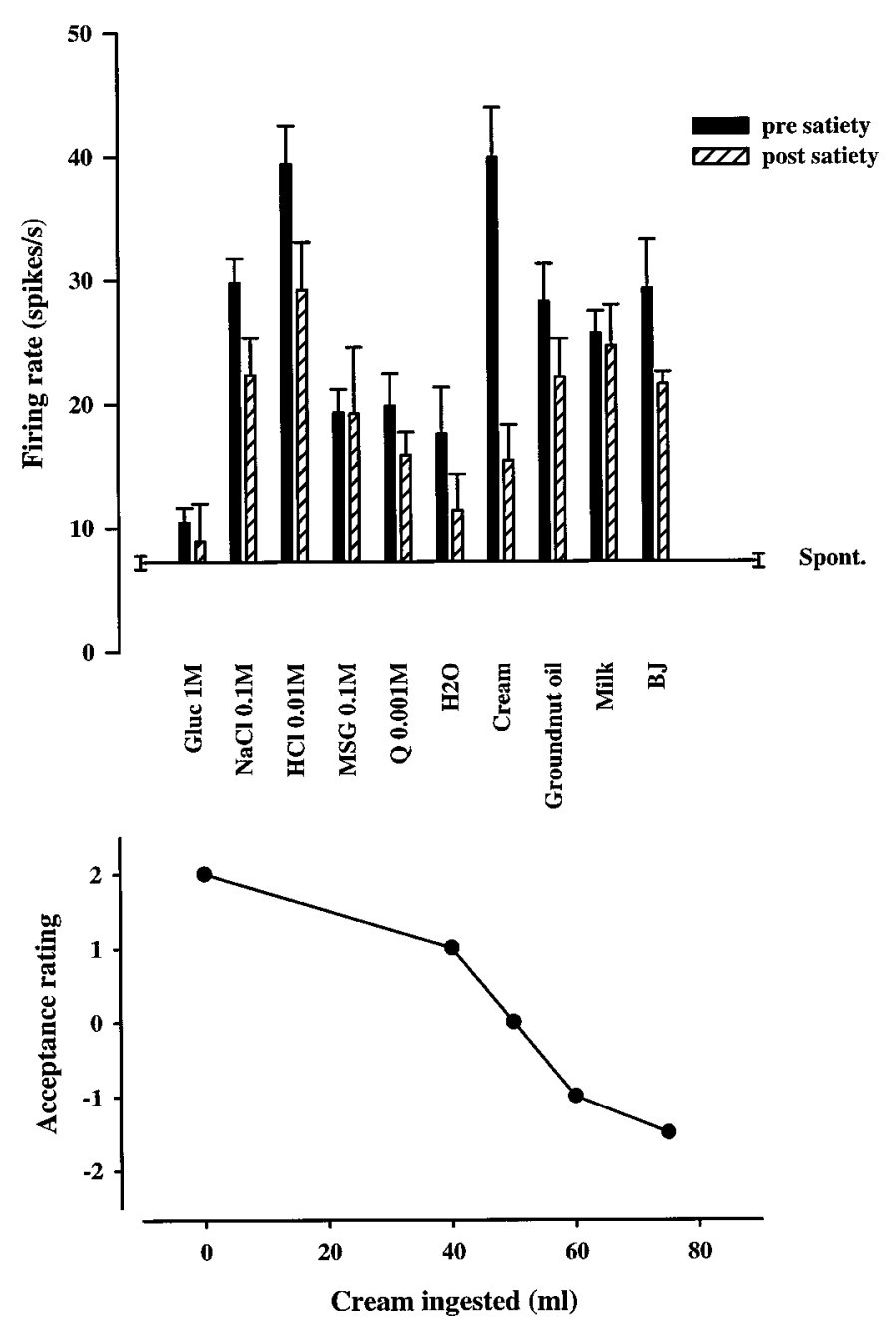

Figure 5. Responses of a primate orbitofrontal cortex neuron to the texture of the fats cream and groundnut oil and to different tastes before and after feeding to satiety with cream. Conventions as in Figures 1, 3, and 4 .

small response to some taste stimuli (e.g., sour, $\mathrm{HCl}$ ). The neuron had no response to a wide range of odors (data not shown). The neuron also significantly increased its firing rate when the monkey was eating peanuts, chocolate, and chocolate-hazelnut spread, and it was found that the firing rate to these foods was higher if they were emulsified to liquid form by blending with a small amount of water. The neuron did not respond significantly when the monkey was eating solid apple, so the increases to eating solid forms of fat were not because of chewing. This investigation indicated that this neuron responded when a solid fatty food is being chewed and eaten, and at the same time indicated that the fat-sensing system in the mouth can produce larger effects on these orbitofrontal neurons when the solid fatty food is made into a more liquid texture, which occurs partly by chewing, and can be produced by emulsification.

A summary of the testing and response properties of the neurons with responses to fat in the mouth [or fat-related stimuli in terms of texture such as silicone oil and paraffin oil in the mouth (or in one case smelled)] is shown in Table 1. The firing rates of the neurons in spikes/sec are shown for the taste stimuli to which the neurons had significant increases in firing rate in the ANOVA and post hoc test. The firing rates of the neurons in

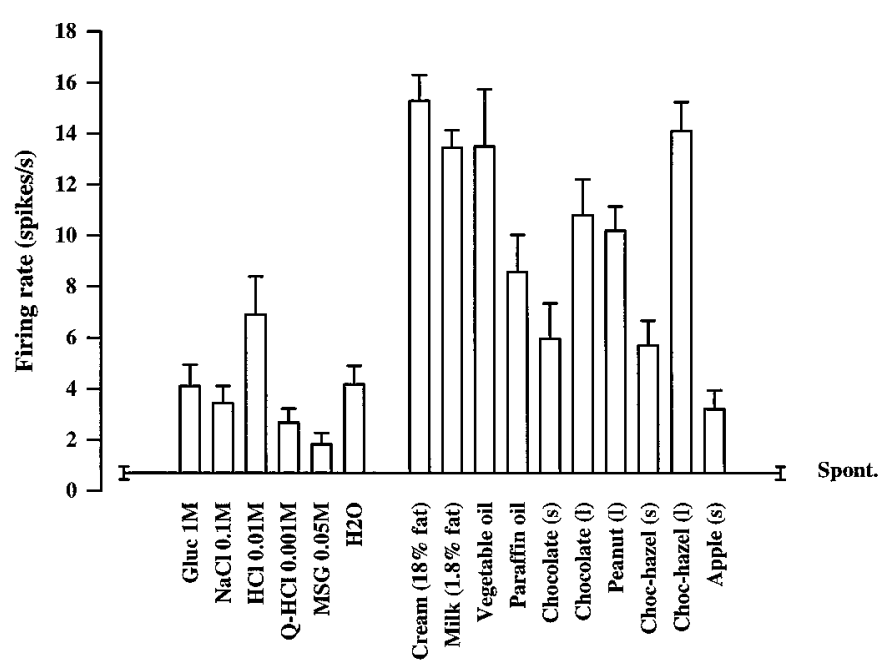

Figure 6. Responses of a primate orbitofrontal cortex neuron (bb027) to fat in the mouth, to stimuli with a similar texture, and to some of the taste stimuli. Conventions as in Figure 1. The neuron also responded when the monkey was eating peanuts, chocolate, and chocolate-hazelnut (Chochazel) spread, with a greater increase of firing rate to each if they were emulsified by blending with a small amount of water $(l)$ compared with when they were solid $(s)$. The responses to all the fat and fat-related stimuli and to $\mathrm{HCl}$ were significantly greater than the spontaneous rate (in the majority of cases, $p<0.001$ ) as shown by a Dunnett's post hoc test after a significant one-way ANOVA $(p<0.001)$. The neuron did not respond when the monkey was eating solid apple.

spikes/sec are shown for all the fat and fat-related stimuli tested. (These firing rates were all significant increases in the post hoc Dunnett's test for each stimulus compared with the spontaneous rate, except where indicated by NS. Even in these latter cases, the responses to pure fat or fat texture stimuli were typically significant in preplanned groupings, as described under the descriptions of the responses of cells be 0511 and be047.) For example, we note that 7 of the 10 neurons that responded to cream in the mouth also responded to at least one of the other fat stimuli or to the combined fat stimulus group. The 11 neurons shown in Table 1 came from a sample of 1145 neurons investigated in the orbitofrontal cortex. Of the nine neurons with responses to fat in the mouth fully tested for both taste and fat-related responses, seven responded to both taste and fat, and two did not respond to taste. Of six neurons tested for whether the effect of eating fat to satiety decreased the response of the neuron to fat, all showed sensoryspecific satiety effects. For one neuron the effect measured was a sensory-specific reduction in the response to the odor of cream with which the monkey had been fed to satiety, and for one neuron the effect measured was to both the sight of cream (to which the neuron responded) and its mouth feel.

\section{Localization of recordings}

The reconstructed positions of the neurons in this study are shown on Figure 7. Neurons responsive to fat are shown by triangles, neurons also shown to receive a taste input are shown by circles, and neurons also shown to receive an olfactory input are indicated by squares. The neurons were located within the orbitofrontal cortical area.

\section{DISCUSSION}

The results described here show that there is a population of neurons in the primate orbitofrontal cortex that responds to the sensory properties of fat in the mouth. The responses of individ- 


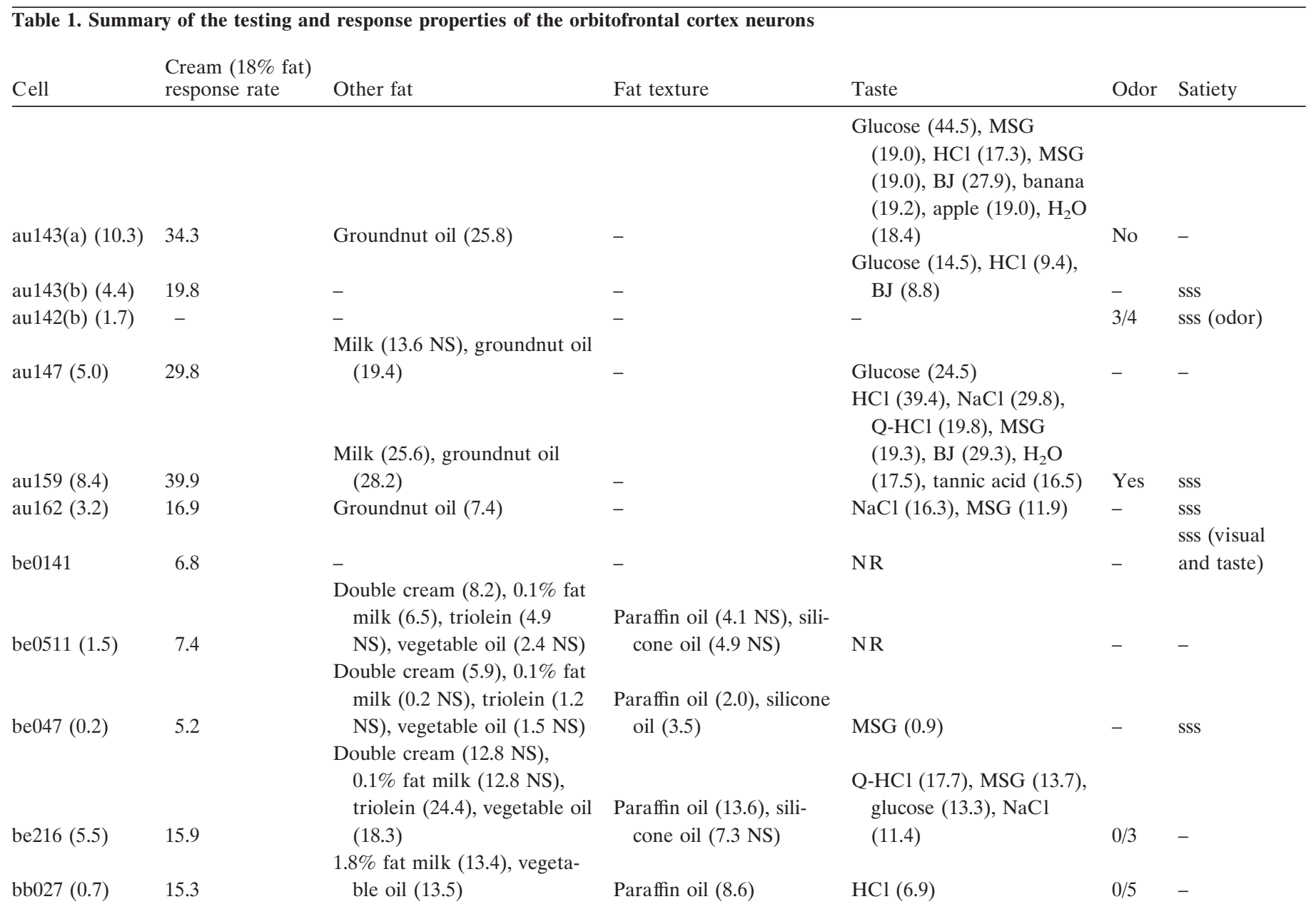

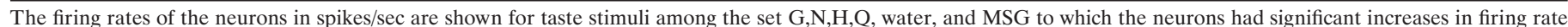

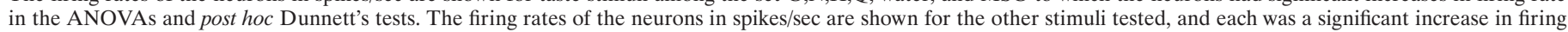

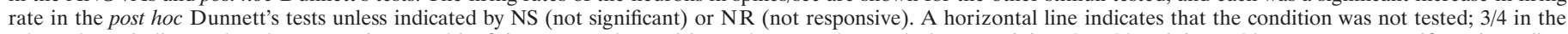

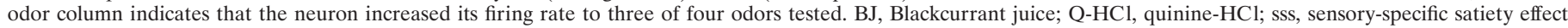
found.

ual neurons to fats were typically highly significant (in the majority of cases with $p \ll 0.001$ ). Although the proportion of neurons responding in this way over the whole sample of neurons is small $(11 / 1145=0.96 \%)$, the actual proportion clearly depends on the extent to which the correct area for the neurons (Fig. 7) has been sampled. Indeed, in the recordings in monkey au, 6 of 320 neurons $(1.9 \%)$ recorded in the orbitofrontal cortex had responses to fat stimuli. The areas in which these neurons were found were in the caudal orbitofrontal cortex, in an area that includes and probably extends medial to the secondary taste cortex as defined by the cortex receiving inputs from the primary taste cortex (Baylis et al., 1994).

The actual proportion of fat-responsive neurons in the orbitofrontal cortical regions that contain representations of the mouth feel of fat is likely to be higher than the proportion found here $(0.96 \%)$, for a number of reasons. First, of the cells isolated, it was not possible to perform sufficient testing on all to prove that they did not respond to fat. (For 4 of 16 cells tested very extensively with fat, it was possible to show that they did not respond to fat.) Second, not all of the recording tracks were necessarily into the main areas (Fig. 7) where neurons with responses to fat were located. Part of the evidence for this is that in one monkey (au, in which the tracks were presumably directed more at these neu- rons), the proportion of cells was 6/320 (1.9\%). We note that although this proportion of neurons responding to fat in the orbitofrontal cortex is low, the proportion of neurons responding to other types of stimuli in this region is also quite low. For example, Rolls and Baylis (1994) found that in a sample of $>2000$ neurons recorded in the orbitofrontal cortex, $\sim 3.5 \%$ responded unimodally to taste, $0.8 \%$ unimodally to olfactory stimuli, $1.5 \%$ unimodally to visual stimuli, $0.8 \%$ to both gustatory and olfactory stimuli, $1.2 \%$ to both gustatory and visual stimuli, and $0.3 \%$ to visual and olfactory stimuli. The more lateral fat-responsive neurons in the orbitofrontal cortex (Fig. 7) were in a region that is defined as secondary taste cortex in that anatomically it receives inputs from the primary taste cortex (Baylis et al., 1994), whereas the more medial neurons were in a more multimodal region in which taste, olfactory, and visual neurons have been recorded (Rolls and Baylis, 1994). Carmichael and Price (1995) have shown anatomically that the more medial region receives inputs directly from the primary somatosensory cortex, and in addition that there is rich interconnectivity between different subregions of the orbitofrontal cortex (Carmichael and Price, 1996). Visual and auditory inputs project directly to several other subregions of the orbitofrontal regions (Barbas, 1993; Carmichael and Price, 1995), but the interconnectivity allows neurons in many areas of 
A
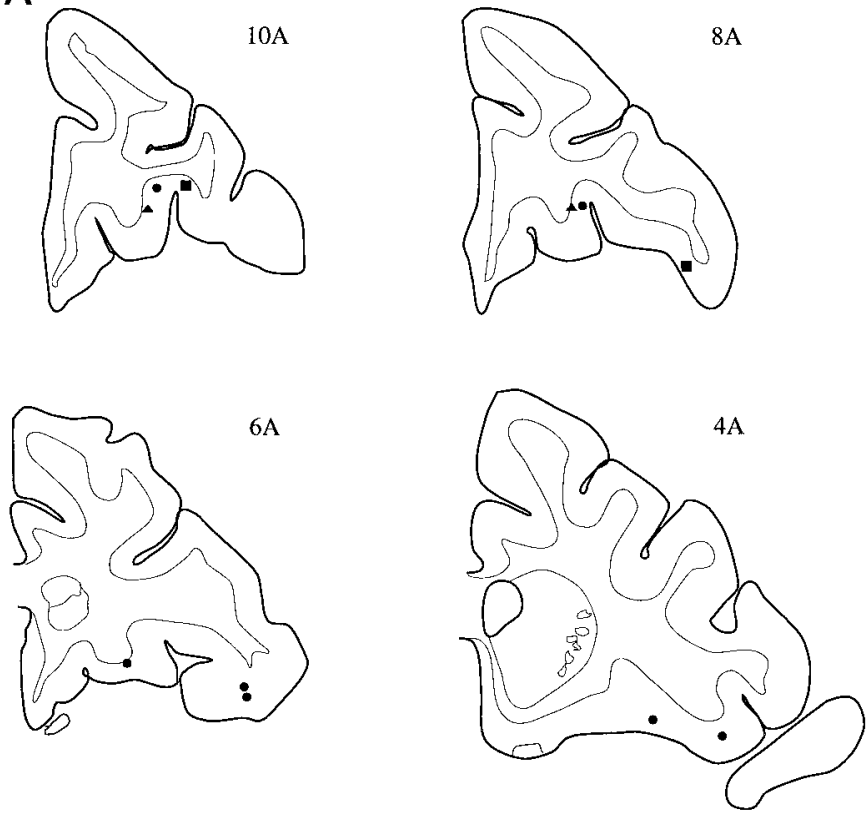

B

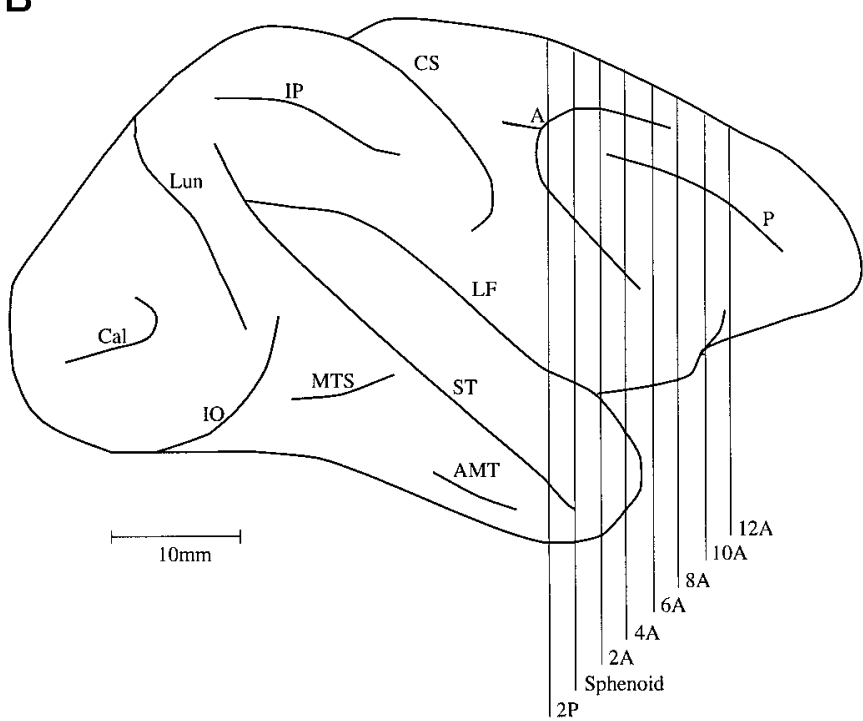

Figure 7. The reconstructed positions of the neurons in this study. $A$, Neurons responsive to fat are shown by triangles, neurons responsive to fat and taste are shown by circles, and fat-related neurons shown to receive an olfactory input are indicated by squares. The neurons were located within the orbitofrontal cortical area. (For one macaque the reconstructions are based on the histology and the x-ray data for every track, and for the other they are based on the x-ray data for every track and a standard atlas calibrated in x-ray coordinates.) $B$, A lateral view of the macaque brain showing the levels with respect to sphenoid of the coronal sections shown in $A$.

the orbitofrontal cortex to respond to inputs from different sensory modalities (Rolls and Baylis, 1994).

Some of the findings provide evidence that the responsiveness of the neurons is sensed by the mouth feel (texture) of fat and not by chemical sensing. First, some of the fat-responsive neurons can be unimodal, responding to the mouth feel of fat and not to taste. Second, of the neurons that respond to fat and taste stimuli, each is tuned to have best responses to a different set of tastes (Figs. 2, $3,4,6)$, so that there is no special "taste" tuning of the fat- responsive neurons that accounts for their responses to fat. Third, when sensory-specific satiety to fat is produced, the responses of the same neuron to taste may be little affected or even increased, so that the responses to fat do not appear to be produced through taste channels (i.e., channels with chemical selectivity). Fourth, all of the neurons tested responded to stimuli that were chemically different from fat but had a similar mouth feel, because this would be sensed through the somatosensory system. These stimuli included silicone oil $\left.\left[\mathrm{Si}\left(\mathrm{CH}_{3}\right)_{2} \mathrm{O}\right)_{n}\right]$ and paraffin oil (hydrocarbon).

The mouth feel of fat appropriate for sensing fat in food is what affects these neurons somewhat selectively, in that the same neurons did not respond when the monkey ate nonfat solid food such as apple, nor when the inside or outside of the mouth was mechanically stimulated with cotton buds, nor when a nonfood object was chewed (such as tubing). Having said this, the data recorded from the neuron shown in Figure 6 showed that this neuron was more affected by fat in a liquified, emulsified form than in a solid form (as shown by the experiments with solid and liquified chocolate, nuts, and chocolate-hazelnut mixture). This seems entirely plausible, in that it is the mouth feel of slickness that tends to help humans identify a food as fat-containing. Although testing with solid forms of fat was performed mainly for this neuron, in one additional neuron (be216) we found that although the neuron did respond to liquid fat, when a specially prepared more solid form of cream was tested (described commercially as extra thick cream), the neuronal firing to this was much less $(5.5$ spikes/sec) than to normal double cream. Taken in the context of the findings with sensory-specific satiety, an implication is that these neurons would be more effectively activated during the eating of a fatty meal if the fat is in liquid form. This more effective activation of these neurons by liquid fat might result in more rapid sensory-specific satiety (because the total sensory stimulation would build up more quickly), resulting in more rapid sensory-specific satiety with fat in liquid than in more solid form. The implication is that eating food slowly and chewing it well (to emulsify it), or taking foods containing fat in a form in which the fat is liquid, would be expected to reduce the total amount of fat consumed. This could have implications for dieting and body weight control. It would be of interest to test these implications as well as to obtain data from additional neurons tested with fats with different physical properties.

It has been suggested that fats might also be detected in the mouth based on free fatty acid sensing by taste cells (Gilbertson et al., 1997). It has been shown that rat taste cells can respond to cis-polyunsaturated fatty acids (such as linoleic acid and arachidonic acid). However, the mechanism is unlikely to account for the effects described here and the oral sensory properties of fats in humans, because (1) the cells described here in primates responded to chemical stimuli that contained no fats or fatty acids (e.g., paraffin oil and silicone oil); (2) salivary lingual lipase, which would be needed to release fatty acids from fat in the mouth, is present in the rat but probably limited in humans (Spielman et al., 1993); and (3) the time course of the fatty acid effect is in the order of minutes (Gilbertson et al., 1997), whereas humans and the cells described here in the primate orbitofrontal cortex respond to the mouth feel of fat very rapidly $(<1 \mathrm{sec})$.

The present results extend the known sensory mechanisms by which sensory-specific satiety can be produced. The present results provide evidence that it can be produced by the mouth feel 
of fat and also by stimuli that have been associated with these by learning (Rolls et al., 1996a), for example the smell or the sight of a fatty food such as cream. In previous experiments at both the behavioral level in humans and monkeys and at the neuronal level in monkeys, it has been shown that sensory-specific satiety can be based on and produced by taste, visual, and olfactory sensory properties of foods (B. J. Rolls et al., 1981a,b, 1982, 1988; E. T. Rolls et al., 1989; Critchley and Rolls, 1996b; Rolls, 1997, 1999a; Rolls and Rolls, 1997).

The findings described here that the neuronal responses to the sensory properties of fat decrease to zero when the monkey is fed to satiety with fat, and that the acceptability of the fat to the monkey at the same time decreases to zero, provide an indication that it is the reward value of the fat that is represented in the primate orbitofrontal cortex. This is entirely consistent with the fact that the primate orbitofrontal cortex supports good brainstimulation reward and that this brain-stimulation reward is hunger dependent (Mora et al., 1979, 1980; Rolls, 1999a). The result is also consistent with the evidence that the pleasantness of touch is represented in the human somatosensory cortex (Rolls et al., 1997; Francis et al., 1999) and that the responses of neurons in the primate orbitofrontal cortex to the sight, smell, and taste of a food all decrease to zero when the monkey is fed to satiety with that food (Rolls et al., 1989; Critchley and Rolls, 1996b). This underlines the importance of the primate orbitofrontal cortex in the representation of food, not only by combining inputs defining a food from different sensory modalities including somatosensory as shown here, but also by representing the reward value of that sensory input (Rolls, 1999a).

In conclusion, the data presented here are the first showing the representation of the sensory properties of fat in cortical neurons in a region where there are taste neurons and, in some cases, within neurons that are responsive to tastes. These findings are important to the study of feeding behavior and body weight control, because almost all previous work on the satiating effects of fats have concentrated on peripheral signaling of fat ingestion through hormonal mediators such as cholecystokinin and leptin (Rolls, 1999a). It now appears that the sensory properties of fatty foods are encoded within populations of cortical taste neurons and these sensory representations of fat are influenced by motivational factors such as hunger and satiety.

\section{REFERENCES}

Aggleton JP, Passingham RE (1981) Syndrome produced by lesions of the amygdala in monkeys (Macaca mulatta). J Comp Physiol Psychol 95:961-977.

Barbas H (1993) Organization of cortical afferent input to orbitofrontal areas in the rhesus monkey. Neuroscience 56:841-864.

Baylis LL, Rolls ET, Baylis GC (1994) Afferent connections of the caudolateral orbitofrontal cortex taste area of the primate. Neuroscience 64:801-812.

Bell EA, Castellanos VH, Pelkman CL, Thorwart ML, Rolls BJ (1998) Energy density of foods affects energy intake in normal-weight women. Am J Clin Nutr 67:412-420.

Benno Y, Honjo S, Mitsuoke T (1987) Effect of two year milk-feeding on the gastrointestinal microflora of the cynomolgus monkey (Macaca fascicularis). Microbiol Immunol 31:943-947.

Carmichael ST, Price JL (1995) Sensory and premotor connections of the orbital and medial prefrontal cortex of macaque monkeys. J Comp Neurol 363:642-664.

Carmichael ST, Price JL (1996) Connectional networks within the or- bital and medial prefrontal cortex of macaque monkeys. J Comp Neurol 371:179-207.

Carmichael ST, Clugnet M-C, Price JL (1994) Central olfactory connections in the macaque monkey. J Comp Neurol 346:403-434.

Critchley HD, Rolls ET (1996a) Olfactory neuronal responses in the primate orbitofrontal cortex: analysis in an olfactory discrimination task. J Neurophysiol 75:1659-1672.

Critchley HD, Rolls ET (1996b) Hunger and satiety modify the responses of olfactory and visual neurons in the primate orbitofrontal cortex. J Neurophysiol 75:1673-1686.

Critchley HD, Rolls ET (1996c) Responses of primate taste cortex neurons to the astringent tastant tannic acid. Chem Senses 21:135-145.

Feigenbaum JD, Rolls ET (1991) Allocentric and egocentric spatial information processing in the hippocampal formation of the behaving primate. Psychobiology 19:21-40.

Francis S, Rolls ET, Bowtell R, McGlone F, O'Doherty J, Browning A, Clare S, Smith E (1999) The representation of the pleasantness of touch in the human brain, and its relation to taste and olfactory areas. NeuroReport, in press.

Gilbertson TA, Fontenot DT, Liu L, Zhang H, Monroe WT (1997) Fatty acid modulation of $\mathrm{K}+$ channels in taste receptor cells: gustatory cues for dietary fat. Am J Physiol 272:C1203-C1210.

Johnson J, Vickers Z (1993) Effects of flavor and macronutrient composition of food servings on liking, hunger and subsequent intake. Appetite 21:25-39.

Merrill EG, Ainsworth A (1972) Glass-coated platinum-plated tungsten microelectrodes. Med Biol Eng Comput 10:662-672.

Mora F, Avrith DB, Phillips AG, Rolls ET (1979) Effects of satiety on self-stimulation of the orbitofrontal cortex in the monkey. Neurosci Lett 13:141-145.

Mora F, Avrith DB, Rolls ET (1980) An electrophysiological and behavioural study of self-stimulation in the orbitofrontal cortex of the rhesus monkey. Brain Res Bull 5:111-115.

Rolls BJ (1995) Carbohydrates, fats, and satiety. Am J Clin Nutr [Suppl] 61:960S-967S.

Rolls BJ, Rolls ET, Rowe EA, Sweeney K (1981a) Sensory-specific satiety in man. Physiol Behav 27:137-142.

Rolls BJ, Rowe EA, Rolls ET, Kingston B, Megson A, Gunary R (1981b) Variety in a meal enhances food intake in man. Physiol Behav 26:215-221.

Rolls BJ, Rowe EA, Rolls ET (1982) How sensory properties of foods affect human feeding behavior. Physiol Behav 29:409-417.

Rolls BJ, Hetherington M, Burley VJ (1988) The specificity of satiety: the influence of foods of different macronutrient content on the development of satiety. Physiol Behav 43:145-153.

Rolls ET (1976) The neurophysiological basis of brain-stimulation reward. In: Brain-stimulation reward (Wauquier A, Rolls ET, eds), pp 65-87. Amsterdam: North Holland.

Rolls ET (1989) Information processing in the taste system of primates. J Exp Biol 146:141-164.

Rolls ET (1994) Neural processing related to feeding in primates. In: Appetite: neural and behavioural bases (Legg CR, Booth DA, eds), pp 11-53. Oxford: Oxford UP.

Rolls ET (1995) Central taste anatomy and neurophysiology. In: Handbook of olfaction and gustation (Doty RL, ed), pp 549-573. New York: Dekker.

Rolls ET (1997) Taste and olfactory processing in the brain and its relation to the control of eating. Crit Rev Neurobiol 11:263-287.

Rolls ET (1999a) The brain and emotion. Oxford: Oxford UP.

Rolls ET (1999b) Taste, olfactory, visual and somatosensory representations of the sensory properties of foods in the brain, and their relation to the control of food intake. In: Neural control of macronutrient selection (Seeley RJ, Berthoud H-R, eds). Boca Raton, FL: CRC, in press.

Rolls ET, Baylis LL (1994) Gustatory, olfactory and visual convergence within the primate orbitofrontal cortex. J Neurosci 14:5437-5452.

Rolls ET, Rolls JH (1997) Olfactory sensory-specific satiety in humans. Physiol Behav 61:461-473.

Rolls ET, Sienkiewicz ZJ, Yaxley S (1989) Hunger modulates the responses to gustatory stimuli of single neurons in the caudolateral orbitofrontal cortex of the macaque monkey. Eur J Neurosci 1:53-60.

Rolls ET, Yaxley S, Sienkiewicz ZJ (1990) Gustatory responses of single 
neurons in the orbitofrontal cortex of the macaque monkey. J Neurophysiol 64:1055-1066.

Rolls ET, Critchley H, Mason R, Wakeman EA (1996a) Orbitofrontal cortex neurons: role in olfactory and visual association learning. J Neurophysiol 75:1970-1981.

Rolls ET, Critchley H, Wakeman EA, Mason R (1996b) Responses of neurons in the primate taste cortex to the glutamate ion and to inosine 5'-monophosphate. Physiol Behav 59:991-1000.

Rolls ET, Francis S, Bowtell R, Browning D, Clare S, Smith E, McGlone F (1997) Pleasant touch activates the orbitofrontal cortex. NeuroImage 5:S17.

Scott TR, Yaxley S, Sienkiewicz ZJ, Rolls ET (1986a) Taste responses in the nucleus tractus solitarius of the behaving monkey. J Neurophysiol 55:182-200.
Scott TR, Yaxley S, Sienkiewicz ZJ, Rolls ET (1986b) Gustatory responses from the frontal opercular cortex of the alert cynomolgus monkey. J Neurophysiol 56:876-890.

Spielman AL, D'Abundo S, Field RB, Schmale H (1993) Protein analysis of human von Ebner saliva and a method for its collection from the foliate papilla. J Dent Res 72:1331-1335.

Streett JW, Jonas AM (1980) Lactose intolerance in the stumptail macaque (Macaca arctoides): case report. Lab Anim Sci 30:80-84.

Warwick ZS, Hall WG, Pappas TN, Schiffman SS (1993) Taste and smell sensations enhance the satiating effect of both a high-carbohydrate and a high-fat meal in humans. Physiol Behav 53:553-563.

Yaxley S, Rolls ET, Sienkiewicz ZJ (1990) Gustatory responses of single neurons in the insula of the macaque monkey. J Neurophysiol 63:689-700. 